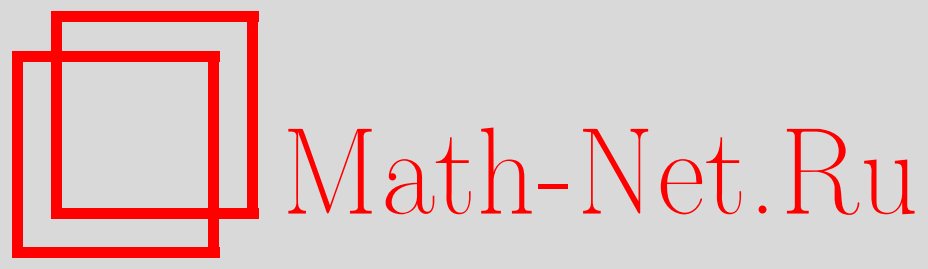

А. М. Будылин, В. С. Буслаев, Квазиклассические асимптотики решений матричной задачи сопряжения с квадратичной осцилляцией внедиагональных элементов, Функи. анализ и его прил., 2014, том 48, выпуск 1, 1-18

DOI: https://doi.org/10.4213/faa3137

Использование Общероссийского математического портала MathNet.Ru подразумевает, что вы прочитали и согласны с пользовательским соглашением http://www . mathnet.ru/rus/agreement

Параметры загрузки:

IP: 54.198 .187 .58

26 апреля 2023 г., 10:47:24

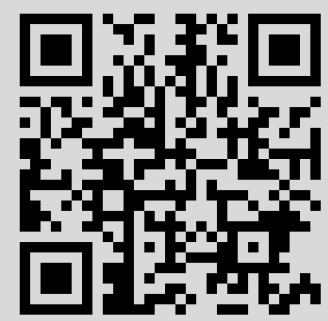




\title{
Квазиклассические асимптотики решений матричной задачи сопряжения с квадратичной осцилляцией внедиагональных элементов
}

\author{
(c) 2014. А. М. Будылин, В. С. БуслАЕВ
}

\begin{abstract}
Исследуется асимптотика решений матричных $(2 \times 2)$-задач сопряжения (задач Римана-Гильберта) с быстро осциллирующими внедиагональными членами и квадратичной фазовой функцией. Используется новый подход к исследованию таких задач на основе идей метода стационарной фазы и теории М. Г. Крейна. Данная задача является модельной для исследования асимптотик решений задач сопряжения с несколькими точками поворота. Найдены полные в степенных порядках асимптотические разложения для решений рассматриваемой задачи. Полученные асимптотики использованы для построения асимптотик решений задачи Коши для нелинейного уравнения Шрёдингера на больших временах.
\end{abstract}

\section{§1. Предварительные замечания}

Постановка задачи. Под задачей сопряжения, или задачей Римана-Гильберта, мы понимаем задачу аналитической факторизации функции на вещественной оси: для заданной функции $V$ вещественного аргумента построить функции $H_{+}, H_{-}$, допускающие аналитическое продолжсение соответственно в верхнюю и ниюнюю полуплоскости комплексной плоскости, такие, что на вещественной оси выполняется равенство

$$
H_{+}=H_{-} V .
$$

В случае матричной задачи сопряжения рассматриваемые здесь функции полагаются матричными. Мы ограничимся рассмотрением случая квадратных матриц второго порядка. Вопросы разрешимости матричных задач сопряжения были изучены М. Г. Крейном. Некоторые аспекты теории Крейна нам понадобятся в вопросах обоснования, и мы коснемся их в свое время.

Пока же отметим, что в сфере наших интересов роль матрицы сопряжения $V$ будет играть матрица вида

$$
V(x)=\left(\begin{array}{cc}
a(x) & b(x) e^{-i t \Phi(x)} \\
c(x) e^{i t \Phi(x)} & d(x)
\end{array}\right)
$$

где фазовая функция $\Phi(x)$ имеет вид $x^{2} / 2$. Более общий случай фазовой функции - гладкой вещественной функции с конечным числом простых стационарных точек - мы рассмотрим в следующей статье.

Функции $a-1, b, c, d-1$ будут предполагаться комплекснозначными из класса Шварца, так что при $x \rightarrow \infty$ матрица $V$ превращается в единичную. Исходя из элементарности задачи факторизации диагональной матрицы можно утверждать, что налагаемые нами ограничения носят достаточно общий характер. На самом деле нам потребуются некоторые более специфические ограничения 
на функции $a, b, c, d$, которые позволят оставить в стороне некоторые специфические вопросы факторизации функций.

Прежде всего, мы будем считать, что на всей оси выполнено условие

$$
\Delta(x) \equiv a(x) d(x)-b(x) c(x) \neq 0,
$$

причем индекс функции $\Delta$ на замкнутой оси равен нулю.

Более того, определим $P$ как характеристическую функцию полуоси $(0,+\infty)$, и пусть $Q=1-P$. Предполагая, что функция $d$ не обращается в нуль на носителе функции $P$, дополним график разрывной функции $a Q+\Delta d^{-1} P$ отрезком прямой, соединяющим точки $a(0)$ и $D(0) d^{-1}(0)$. Мы будем считать, что полученная непрерывная кривая также имеет нулевой индекс (в частности, функция $a$ не обращается в нуль на носителе функции $Q)$. Напомним, что индекс только что описанной непрерывной кривой называют индексом разрывной функции $a Q+\Delta d^{-1} P$.

Переменная $t$ будет играть роль большего положительного параметра. Нас будет интересовать асимптотическое поведение факторизационных множителей $H_{ \pm}$при $t \rightarrow+\infty$ при естественном условии нормировки

$$
H_{ \pm}(x) \underset{x \rightarrow \infty}{\longrightarrow} I
$$

где $I$ - единичная матрица.

Краткая история вопроса. Интерес к задачам сопряжения описанного типа в значительной степени был обусловлен их приложениями к исследованию известных нелинейных уравнений математической физики, таких, как нелинейное уравнение Шрёдингера (НУШ) или уравнение Кортевега-де Фриза, т. е. тех, которые допускают явные решения спектральными методами. Первоначальные отношения между нелинейными уравнениями и спектральными объектами были сформулированы как раз в терминах соответствующих матричных задач сопряжения для аналитических функций.

Изучению асимптотического поведения решений нелинейных уравнений на больших временах посвящена обширная литература. Здесь нам бы хотелось отметить подход Итса [5] и его развитие в исследованиях [6], [7] Дейфта и Жу.

Наши исследования [2], [3] по нелинейным уравнениям первоначально велись в рамках так называемого прямого подхода, основанного на исследовании соответствующих уравнений Гельфанда-Левитана-Марченко. Прямой подход имеет свои преимущества, в частности, он не зависит от полной интегрируемости системы. Однако у него есть и определенные слабости. Дело в том, что хотя ответ легко выписывается в терминах конечной комбинации ядер некоторых интегральных операторов, его зависимость от асимптотических параметров не вполне прозрачна, и требуются дополнительные достаточно трудоемкие вычисления.

Напротив, характерные зависимости от асимптотических параметров в подходе Итса-Дейфта-Ку возникают весьма естественно. Однако реализация такого подхода требует деформаций первоначального контура к целой системе сложных контуров с последующим решением на каждом из них соответствующей задачи Римана-Гильберта.

Анализ сложившейся ситуации привел нас к некоторой новой схеме асимптотического решения матричных задач сопряжения, свободной от упомянутых недостатков. Если подход Дейфта-Жу можно ассоциировать с идеями метода 
перевала для вычисления асимптотик интегралов, то наш подход естественно ассоциировать с идеями метода стационарной фазы. Признавая, что мы не первые среди тех, кто апеллирует к методу стационарной фазы при исследовании описанной выше задачи, мы должны отметить, что нам не удалось найти сколько-нибудь законченной работы в этом направлении.

Общая характеристика ответа и формулировка результата. Главный вклад в асимптотику при $t \rightarrow \infty$ множителей $H_{ \pm}$вносит стационарная точка фазовой функции $\Phi$. Отметим, что случай одной стационарной точки не только самый простой, но также, по существу, это модель при анализе общего случая нескольких простых стационарных точек.

Для этого случая мы не только выписываем старший член асимптотики решения, но строим полные в степенных порядках (но не степенные) асимптотические разложения.

Для предварительной формулировки результата мы введем в рассмотрение пространство гёльдеровых функций $H^{\mu, \theta}(\mathbb{R})$ с нормой

$$
\|f\|_{\mu, \theta}=\sup _{x, h \in \mathbb{R}}(1+|x|)^{\theta}\left(|f(x)|+\frac{|f(x+h)-f(x)|}{|h|^{\mu}}\right) .
$$

В условиях на матрицу $V$, описанных выше, мы делаем следующее.

- Мы доказываем, что при достаточно больших $t$ решение $H_{ \pm}$задачи сопряжения (1)-(2) существует, и вычисляем асимптотику $H_{ \pm}^{0}$ решения, которая за вычетом единичной матрицы принадлежит пространству $H^{\mu, 1}(\mathbb{R})$ при достаточно малом $\mu>0$.

- Мы описываем простую итерационную процедуру, которая позволяет получить асимптотику решений с любой степенью точности в степенных порядках в пространстве $H^{\mu, 1}(\mathbb{R})$.

- Мы также показываем, что асимптотика решений может быть охарактеризована разложениями вида

$$
H_{ \pm}(x)-I \sim t^{ \pm i \nu_{0}} \sum_{\substack{k \geqslant 0 \\ 0 \leqslant j \leqslant k}} h_{k j \pm}(x, t) t^{-k / 2} \ln ^{j} t+t^{\mp i \nu_{0}} \sum_{\substack{k \geqslant 1 \\ 0 \leqslant j \leqslant k}} \tilde{h}_{k j \pm}(x, t) t^{-k / 2} \ln ^{j} t
$$

где $h_{k j \pm}, \tilde{h}_{k j, \pm}$ - функции переменной $x, L_{2}(\mathbb{R})$-норма которых ограничена относительно параметра $t$.

Наконец, мы применяем полученные результаты для вычисления асимптотик решений задачи Коши для НУШ на больших временах.

\section{§2. Задача сопряжения с квадратичной осцилляцией}

2.1. Предварительный анализ. Наводящие соображения. Важнейшая мотивирующая идея может быть описана следующим образом: хорошо известно, что функция $g(x)=f(x) e^{i t x}$, где $f-$ произвольная гладкая достаточно быстро исчезающая на бесконечности функция, является почти аналитической в $\mathbb{C}_{+}$ при $t \rightarrow+\infty$. При этом имеется в виду следующее асимптотическое соотношение:

$$
p g \underset{t \rightarrow+\infty}{\sim} g, \quad(p g)(x)=\frac{-1}{2 \pi i} \int_{\mathbb{R}} \frac{g(y) d y}{x-y+i 0},
$$


так что оператор $p$ является оператором ортогонального проектирования в $L_{2}(\mathbb{R})$ на подпространство функций, аналитических в верхней комплексной полуплоскости.

Точно таким же образом функция $g$ является почти аналитической в $\mathbb{C}_{-}$при $t \rightarrow-\infty$ :

$$
q g \underset{t \rightarrow-\infty}{\sim} g, \quad(q g)(x)=\frac{1}{2 \pi i} \int_{\mathbb{R}} \frac{g(y) d y}{x-y-i 0} .
$$

Оператор $q$ является оператором ортогонального проектирования на подпространство функций в $L_{2}(\mathbb{R})$, допускающих аналитическое продолжение в нижнюю комплексную полуплоскость. Хорошо известно, что $p+q=I$.

Приложение $\kappa$ квадратичной экспоненте. В нашем случае быстрая осцилляция порождается квадратичной экспонентой. Это приводит к естественной необходимости модифицировать описанные выше соображения. А именно, в отношении функции $g=f T$ мы по прежнему можем написать $p g \underset{t \rightarrow+\infty}{\sim} g$, только если гладкая функция $f$ имеет носитель на полуоси $(0,+\infty)$. И в этом же случае $q g \underset{t \rightarrow-\infty}{\sim} g$. Наоборот, $p g \underset{t \rightarrow-\infty}{\sim} g$ и $q g \underset{t \rightarrow+\infty}{\sim} g$, если носитель функции $f$ лежит на отрицательной полуоси.

Конечно, все дело в том, что квадратичная экспонента $T=e^{i t x^{2} / 2}$ при $t>0$ продолжается как убывающая функция переменной $x$ в первый и третий квадранты комплексной плоскости $x$, а при $t<0-$ во второй и четвертый.

Эти замечания позволяют весьма просто получить аналитическую факторизацию рассматриваемой матрицы сопряжения соответственно для первогочетвертого и второго-третьего квадрантов комплексной плоскости. Мы опишем эти процедуры ниже более подробно. Сразу отметим, что для решения задачи факторизации на оси нам понадобится сшивка этих процедур. Эта сшивка потребует привлечения некоторой модельной задачи, решение которой базируется на известных аспектах аналитической теории дифференциальных уравнений.

Эффективная факторизаиия на полуоси. Перейдем от матрицы сопряжения $V$ к матрице

$$
W=\left(\begin{array}{cc}
1 & b_{1} T^{-1} \\
0 & 1
\end{array}\right)\left(\begin{array}{cc}
1 & 0 \\
c_{1} T & 1
\end{array}\right)
$$

В соответствии с предыдущими замечаниями мы намерены трактовать данное представление как асимптотически эффективную аналитическую факторизацию $W=W_{-} W_{+}$при $x>0$ и $t \rightarrow+\infty$. Обратим внимание на тот факт, что квадратичные экспоненты в правой части равенства (6) допускают аналитическое продолжение соответственно в четвертый и первый квадранты комплексной плоскости как ограниченные функции.

Упомянутый переход от $V$ к $W$ мы можем осуществить при помощи умножения матрицы $V$ на аналитические в соответствующих полуплоскостях обкладки: $W=\Theta_{-} V \Theta_{+}$, где $\Theta_{-}=\operatorname{diag}\left(\alpha_{-}, \beta_{-}\right), \Theta_{+}=\operatorname{diag}\left(\alpha_{+}, \beta_{+}\right)-$диагональные матрицы, а $\alpha_{ \pm}, \beta_{ \pm}$- функции, которые однозначно определяются при соответствующих предположениях аналитическими факторизационными соотношениями $\beta_{+} \beta_{-} d=1, \alpha_{+} \alpha_{-}(a d-b c)=d$ и единичной асимптотикой на бесконечности. Действительно, как легко видеть, матрица $W$ примет вид

$$
W=\left(\begin{array}{cc}
1+b_{1} c_{1} & b_{1} T^{-1} \\
c_{1} T & 1
\end{array}\right), \quad b_{1}=\alpha_{-} \beta_{+} b, \quad c_{1}=\alpha_{+} \beta_{-} c
$$


и может быть записана как произведение (6).

Для того чтобы предъявить аналогичное разложение для $x<0$, мы должны перейти от матрицы $V$ к матрице $W$ вида

$$
W=\left(\begin{array}{cc}
1 & 0 \\
c_{2} T & 1
\end{array}\right)\left(\begin{array}{cc}
1 & b_{2} T^{-1} \\
0 & 1
\end{array}\right)
$$

который на прежних основаниях мы можем трактовать как асимптотически эффективную аналитическую факторизацию, но уже при $x<0$.

Для этого перехода опять достаточно провести умножение матрицы $V$ на обкладки $\Theta_{ \pm}$, если переопределить функции $\alpha_{ \pm}, \beta_{ \pm}$, полагая, что $\alpha_{+} \alpha_{-} a=1$, $\beta_{+} \beta_{-}(a d-b c)=a$, по-прежнему с единичной асимптотикой на бесконечности. При этом $c_{2}=\alpha_{+} \beta_{-} c, b_{2}=\alpha_{-} \beta_{+} b$.

Вспомогательные скалярные задачи сопряюения. Для того чтобы соединить вместе оба случая, мы определяем функции $\alpha_{ \pm}, \beta_{ \pm}$как решения скалярных задач Римана

$$
\begin{gathered}
\alpha_{+} \alpha_{-}=d \Delta^{-1} P+a^{-1} Q, \quad \beta_{+} \beta_{-}=d^{-1} P+a \Delta^{-1} Q, \quad \Delta=a d-b c \\
\alpha_{ \pm}(x), \beta_{ \pm}(x) \underset{x \rightarrow \infty}{\longrightarrow} 1
\end{gathered}
$$

где $P$ и $Q$ являются характеристическими функциями полуосей $(0, \infty)$ и $(-\infty, 0)$ соответственно. Напомним, что по предположению функции $\Delta$ и $a Q+\Delta d^{-1} P$ имеют нулевой индекс на оси. В этом случае решения задач сопряжения (7) существуют и определены однозначно, см., например, [4]. Мы опишем эти решения явно в следующем разделе. Отметим, что равенство $\left(\alpha_{+} \beta_{+}\right)\left(\alpha_{-} \beta_{-}\right)=\Delta^{-1}$ определяет аналитическую факторизацию на оси гладкой функции $\Delta^{-1}$. При этом на объединении полуосей имеет место разложение

$$
W=\left(\begin{array}{cc}
1 & \gamma T^{-1} P \\
\delta T Q & 1
\end{array}\right)\left(\begin{array}{cc}
1 & \gamma T^{-1} Q \\
\delta T P & 1
\end{array}\right), \quad \gamma=\alpha_{-} \beta_{+} b, \quad \delta=\alpha_{+} \beta_{-} c .
$$

Если бы множители в правой части матричного равенства (8), назовем их $W_{l}$ и $W_{r}$, были эффективно аналитическими в соответствующих полуплоскостях, цель была бы достигнута. Однако данное представление матрицы $W$ как приближение к аналитической факторизации заведомо не пригодно в нуле, поскольку функции сопряжения вспомогательных скалярных задач имеют в нуле скачок. Для коррекции данного разложения из каждого множителя в правой части равенства (8) мы хотим извлечь его асимптотику в нуле (при $x=0)$ и произведение $W_{0}$ полученных асимптотик подвергнуть точной аналитической факторизации $Y_{-} Y_{+}$. Оставшиеся дополнительные множители, которые мы описываем как окаймляющие $L$ и $R$, окажутся эффективно аналитическими в соответствующих комплексных полуплоскостях. Схематично эта процедура представляется переходами $V \mapsto W=W_{l} W_{r}=L W_{0} R=L\left(Y_{-} Y_{+}\right) R=\left(L Y_{-}\right)\left(Y_{+} R\right)$ и описывается ниже более подробно. Следует отметить, что идея использования «верхне-нижней» треугольной факторизации исходной матрицы сопряжения была впервые использована в работах [6], [7], хотя реализация этой идеи отличается от описанной выше.

2.2. Метод стационарной фазы. Bспомогательные асимптотики. В связи с условиями на индекс функций $\Delta$ и $a Q+\Delta d^{-1} P$ достаточно обработать скачок функции $a Q+\Delta d^{-1} P$ в нуле. Положим $\nu_{0}=\frac{-1}{2 \pi} \ln \left(a_{0} d_{0} \Delta_{0}^{-1}\right), a_{0}=a(0)$, 
$b_{0}=b(0), c_{0}=c(0), d_{0}=d(0), \Delta_{0}=\Delta(0)$. Отметим, что, согласно определению индекса разрывной функции, мнимая часть числа $\nu_{0}$ по модулю меньше одной второй: $\left|\operatorname{Im} \nu_{0}\right|<1 / 2$. При этом, с использованием хорошо известных свойств сингулярных интегралов, получаем асимптотическое разложение

$$
\ln \alpha_{+}(x) \underset{x \rightarrow 0}{\sim}-i \nu_{0} \ln (x+i 0)+A_{+}+\sum_{\substack{k \geqslant 1 \\ 0 \leqslant j \leqslant k}} A_{k j}^{+} x^{k} \ln ^{j}(x+i 0) .
$$

Здесь $\ln (x+i 0)$ - главная ветвь логарифма с разрезом по положительной полуоси. Аналогично,

$$
\ln \alpha_{-}(x) \underset{x \rightarrow 0}{\sim} i \nu_{0} \ln (x-i 0)+A_{-}+\sum_{\substack{k \geqslant 1 \\ 0 \leqslant j \leqslant k}} A_{k j}^{-} x^{k} \ln ^{j}(x-i 0),
$$

а также

$$
\ln \beta_{ \pm}(x) \underset{x \rightarrow 0}{\sim} \pm i \nu_{0} \ln (x \pm i 0)+B_{ \pm}+\sum_{\substack{k \geqslant 1 \\ 0 \leqslant j \leqslant k}} B_{k j}^{ \pm} x^{k} \ln ^{j}(x \pm i 0),
$$

знаки согласованы. Мы позволим себе не выписывать явные интегральные представления для констант $A_{ \pm}, A_{k j}^{ \pm}, B_{ \pm}, B_{k j}^{ \pm}$, принимая данные соотношения за их определение. Таким образом, $\alpha_{ \pm}(x) \underset{x \rightarrow 0}{\sim} \sigma_{ \pm}(x)=(x \pm i 0)^{\mp i \nu_{0}} e^{A_{ \pm}}$и $\beta_{ \pm}(x) \underset{x \rightarrow 0}{\sim}$ $\tau_{ \pm}(x)=(x \pm i 0)^{ \pm i \nu_{0}} e^{B_{ \pm}}$, где $(x \pm i 0)^{ \pm i \nu_{0}}=e^{ \pm i \nu_{0} \ln (x \pm i 0)}$, и функции $\ln (x \pm i 0)$ являются главными ветвями логарифма с разрезом по положительной полуоси. Заметим, что $\sigma_{+} \sigma_{-}=d_{0} \Delta_{0}^{-1} P+a_{0}^{-1} Q$ и $\tau_{+} \tau_{-}=d_{0}^{-1} P+a_{0} \Delta_{0}^{-1} Q$. Как следствие $\gamma(x) \underset{x \rightarrow 0}{\sim} \gamma_{0}(x)$, где $\gamma_{0}=b_{0} \sigma_{-} \tau_{+}=b_{0}(x-i 0)^{i \nu_{0}}(x+i 0)^{i \nu_{0}} e^{A_{-}+B_{+}}$и $\delta_{0}=c_{0} \sigma_{+} \tau_{-}=b_{0}(x-i 0)^{-i \nu_{0}}(x+i 0)^{-i \nu_{0}} e^{A_{+}+B_{-}}$. Положим далее $\gamma_{\infty}=\gamma-\gamma_{0}$ и $\delta_{\infty}=\delta-\delta_{0}$.

Поведение функиий $\gamma_{\infty}, \delta_{\infty}$. Функции $\gamma_{\infty}, \delta_{\infty}$ являются гладкими при $x \neq 0$. При $x \rightarrow \pm \infty$ эти функции быстро выходят на степенное поведение с показателями $2 i \nu_{0}$ и $-2 i \nu_{0}$ соответственно, вещественная часть которых по модулю меньше единицы. Наконец, при $x \rightarrow 0$ наши функции обращаются в нуль. Их асимптотическое поведение при $x \rightarrow \pm 0$ контролируется разложениями вида

$$
\gamma_{\infty} \sim x^{2 i \nu_{0}} \sum_{\substack{k \geqslant 1 \\ 0 \leqslant j \leqslant k}} \gamma_{k j}^{ \pm}|x|^{k} \ln ^{j}|x|, \quad \delta_{\infty} \sim x^{-2 i \nu_{0}} \sum_{\substack{k \geqslant 1 \\ 0 \leqslant j \leqslant k}} \delta_{k j}^{ \pm}|x|^{k} \ln ^{j}|x|,
$$

что сразу следует из выписанных выше разложений логарифмов функций $\alpha_{ \pm}$, $\beta_{ \pm}$.

Теорема 2.1. Функиии $\gamma_{\infty} u \delta_{\infty}$ удовлетворяют соотношениям следующего вида. При $t \rightarrow \infty$ справедливы асимптотические разложсения

$$
\begin{aligned}
& p\left(\gamma_{\infty} T^{-1} P\right)=t^{-i \nu_{0}} \sum_{\substack{k \geqslant 1 \\
0 \leqslant j \leqslant k}} \varphi_{k j+}(\sqrt{t} x) t^{-k / 2} \ln ^{j} t+O\left(t^{-\infty}(1+|x|)^{-1}\right), \\
& q\left(\gamma_{\infty} T^{-1} Q\right)=t^{-i \nu_{0}} \sum_{\substack{k \geqslant 1 \\
0 \leqslant j \leqslant k}} \varphi_{k j-}(\sqrt{t} x) t^{-k / 2} \ln ^{j} t+O\left(t^{-\infty}(1+|x|)^{-1}\right),
\end{aligned}
$$




$$
\begin{aligned}
& p\left(\delta_{\infty} T Q\right)=t^{i \nu_{0}} \sum_{\substack{k \geqslant 1 \\
0 \leqslant j \leqslant k}} \psi_{k j-}(\sqrt{t} x) t^{-k / 2} \ln ^{j} t+O\left(t^{-\infty}(1+|x|)^{-1}\right), \\
& q\left(\delta_{\infty} T P\right)=t^{i \nu_{0}} \sum_{\substack{k \geqslant 1 \\
0 \leqslant j \leqslant k}} \psi_{k j+}(\sqrt{t} x) t^{-k / 2} \ln ^{j} t+O\left(t^{-\infty}(1+|x|)^{-1}\right),
\end{aligned}
$$

где коэффициенты разложений удовлетворяют оченкам

$$
\left|\varphi_{k j \pm}(x)\right| \leqslant C_{k j \pm}(1+|x|)^{-1}, \quad\left|\psi_{k j \pm}(x)\right| \leqslant C_{k j \pm}(1+|x|)^{-1} .
$$

При $k \geqslant 2$ функиии $\varphi_{k j \pm}, \psi_{k j \pm}$ дифференцируемы, причем

$$
\left|\varphi_{k j \pm}^{\prime}(x)\right| \leqslant C_{k j \pm}(1+|x|)^{-2}, \quad\left|\psi_{k j \pm}^{\prime}(x)\right| \leqslant C_{k j \pm}(1+|x|)^{-2} .
$$

Функиии $\varphi_{1 j \pm}, \psi_{1 j \pm}$ обладают этими свойствами лишь вне некоторой окрестности нуля. Однако в окрестности нуля они гёлъдеровы. А именно, их норма в пространстве $H^{\mu}$ ограничена при $\mu+|\operatorname{Re}(2 i \nu)|<1$.

Погрешности в разложениях являются гладкими функииями, которые вместе с производными мажсорируются величинами вида $C_{N} t^{-N}(1+|x|)^{-1}$ при произвольном выборе $N$.

Доказательство теоремы мы вынесем в приложение А.1. Как следствия отметим оценки

$$
\begin{aligned}
p\left(\gamma_{\infty} T^{-1} P\right) & =O\left(t^{-1 / 2-i \nu_{0}} \ln t \cdot(1+|x| \sqrt{t})^{-1}\right), \\
p\left(\delta_{\infty} T Q\right) & =O\left(t^{-1 / 2-i \nu_{0}} \ln t \cdot(1+|x| \sqrt{t})^{-1}\right)
\end{aligned}
$$

и, аналогично,

$$
\begin{aligned}
q\left(\gamma_{\infty} T^{-1} Q\right) & =O\left(t^{-1 / 2+i \nu_{0}} \ln t \cdot(1+|x| \sqrt{t})^{-1}\right), \\
q\left(\delta_{\infty} T P\right) & =O\left(t^{-1 / 2+i \nu_{0}} \ln t \cdot(1+|x| \sqrt{t})^{-1}\right) .
\end{aligned}
$$

Положим

$$
L=\left(\begin{array}{cc}
1 & \gamma_{\infty} T^{-1} P \\
\delta_{\infty} T Q & 1
\end{array}\right), \quad R=\left(\begin{array}{cc}
1 & \gamma_{\infty} T^{-1} Q \\
\delta_{\infty} T P & 1
\end{array}\right), \quad L_{-}=q(L), \quad R_{+}=p(R) .
$$

Ввиду оценок (16)-(17) матрица $L$ является почти аналитической в $\mathbb{C}_{-}$, а матрица $R$ почти аналитична в $\mathbb{C}_{+}$, так что с точностью до величин порядка $O\left(t^{-\frac{1}{2} \pm i \nu_{0}} \ln t \cdot(1+|x| \sqrt{t})^{-1}\right)$ справедливы равенства $L \approx L_{-}, R \approx R_{+}$.

Отделение почти аналитических множителей. Используя свойства треугольных матриц, мы легко запишем

$$
\left(\begin{array}{cc}
1 & \gamma T^{-1} P \\
\delta T Q & 1
\end{array}\right)=L\left(\begin{array}{cc}
1 & \gamma_{0} T^{-1} P \\
\delta_{0} T Q & 1
\end{array}\right)
$$

и

$$
\left(\begin{array}{cc}
1 & \gamma T^{-1} Q \\
\delta T P & 1
\end{array}\right)=\left(\begin{array}{cc}
1 & \gamma_{0} T^{-1} Q \\
\delta_{0} T P & 1
\end{array}\right) R
$$

Если данные почти аналитические множители $L$ и $R$ заменить на соответствующие аналитические $L_{-}$и $R_{+}$, мы, в соответствии с оценками (16)-(17), внесем ошибку порядка $O\left(t^{\left|\operatorname{Re}\left(i \nu_{0}\right)\right|-1 / 2} \ln t \cdot(1+\sqrt{t}|x|)^{-1}\right)$. Эта ошибка может быть нивелирована методом невязок, см. разд. 2.5 . 
Обратный ход. Рассмотрим произведение множителей, отвечающих за асимптотику в нуле:

$$
W_{0}=\left(\begin{array}{cc}
1 & \gamma_{0} T^{-1} P \\
\delta_{0} T Q & 1
\end{array}\right)\left(\begin{array}{cc}
1 & \gamma_{0} T^{-1} Q \\
\delta_{0} T P & 1
\end{array}\right)=\left(\begin{array}{cc}
1+\gamma_{0} \delta_{0} P & \gamma_{0} T^{-1} \\
\delta_{0} T & 1+\gamma_{0} \delta_{0} Q
\end{array}\right)=\Omega_{-} V_{0} \Omega_{+},
$$

где

$$
\Omega_{ \pm}=\left(\begin{array}{cc}
\sigma_{ \pm} & 0 \\
0 & \tau_{ \pm}
\end{array}\right), \quad V_{0}=\left(\begin{array}{cc}
a_{0} & b_{0} T^{-1} \\
c_{0} T & d_{0}
\end{array}\right)
$$

Данное представление приводит нас к модельной матричной задаче сопряжения с матричной функцией сопряжения $V_{0}$. Эта модельная задача не содержит медленной зависимости и должна быть решена точно.

2.3. Модельная задача. Постановка модельной задачи сопряжения. Итак, модельная матричная задача сопряжения имеет вид $G_{+}=G_{-} V_{0}$. Решение этой задачи, по существу, завершит наши построения.

Хорошо известно, что данная задача имеет явные решения. Мы должны лишь выбрать решения с правильным асимптотическим поведением. Это поведение выявляется следующими наблюдениями. Произведение матриц

$$
\left(\begin{array}{cc}
1 & \gamma_{\infty} T^{-1} P \\
\delta_{\infty} T Q & 1
\end{array}\right) \Omega_{-} G_{-}^{-1}
$$

асимптотически равное $H_{-}^{-1}$, при $x \rightarrow \infty$ должно стремится к единице. Ввиду того, что на бесконечности $\gamma_{\infty} \sim-\gamma_{0}$ и $\delta_{\infty} \sim-\delta_{0}$, в самом грубом приближении получаем

$$
\Omega_{-} G_{-}^{-1} \sim\left(\begin{array}{cc}
1 & \gamma_{0} T^{-1} P \\
\delta_{0} T Q & 1
\end{array}\right), \quad \text { т. е. } G_{-} \sim\left(\begin{array}{cc}
\sigma_{-} & -\tau_{-} \gamma_{0} T^{-1} P \\
-\sigma_{-} \delta_{0} T Q & \tau_{-}
\end{array}\right) .
$$

Несмотря на то, что это соотношение по понятным причинам не может рассматриваться как асимптотическое для $\left(G_{-}\right)_{12}$ при $x \rightarrow-\infty$ (при его выводе мы использовали самое грубое представление о поведении $H_{-}$на бесконечности), оно позволяет заключить, что компонента $\left(G_{-}\right)_{12}$ решения модельной задачи должна достаточно быстро исчезать при $x \rightarrow-\infty$. Аналогично мы заключаем, что элемент $\left(G_{-}\right)_{21}$ должен достаточно быстро исчезать при $x \rightarrow+\infty$.

Решение моделъной задачи. Решения данной модельной задачи хорошо известны благодаря ее связям с обыкновенным дифференциальным уравнениям для гармонического осциллятора. Тот факт, что матрицы типа $V_{0}$ допускают явную аналитическую факторизацию в функциях параболического цилиндра, был впервые отмечен и использован в работе [5]. Напомним эти связи и опишем решения. Полагая $t=1$, перепишем матрицу $V_{0}$ в виде $V_{0}=e^{-i \frac{x^{2}}{4} \sigma_{3}} U e^{i \frac{x^{2}}{4} \sigma_{3}}$, где $\sigma_{3}=\operatorname{diag}(1,-1)-$ матрица Паули и $U=\left(\begin{array}{ll}a_{0} & b_{0} \\ c_{0} & d_{0}\end{array}\right)$. Тогда $G_{+} e^{-i \frac{x^{2}}{4} \sigma_{3}}=$ $G_{-} e^{-i \frac{x^{2}}{4} \sigma_{3}} U$ и как следствие

$$
G_{+}^{\prime} e^{-i \frac{x^{2}}{4} \sigma_{3}}-i \frac{x}{2} G_{+} \sigma_{3} e^{-i \frac{x^{2}}{4} \sigma_{3}}=\left(G_{-}^{\prime} e^{-i \frac{x^{2}}{4} \sigma_{3}}-i \frac{x}{2} G_{-} \sigma_{3} e^{-i \frac{x^{2}}{4} \sigma_{3}}\right) U .
$$

Сопоставление полученных факторизационных соотношений ведет к следующему заключению:

$$
G_{ \pm}^{\prime}-i \frac{x}{2} G_{ \pm} \sigma_{3}=Z G_{ \pm}
$$


где $Z$ - целая матричная функция. Заметим, что в силу (20) при $x \rightarrow-\infty$

$$
G_{-} \sim\left(\begin{array}{cc}
O\left(x^{i \nu_{0}}\right) & o\left(x^{i \nu_{0}}\right) \\
O\left(x^{-i \nu_{0}}\right) & O\left(x^{-i \nu_{0}}\right)
\end{array}\right) \quad \text { и } \quad G_{-}^{\prime} \sim\left(\begin{array}{cc}
O\left(x^{i \nu_{0}-1}\right) & o\left(x^{i \nu_{0}+1}\right) \\
O\left(x^{-i \nu_{0}+1}\right) & O\left(x^{-i \nu_{0}-1}\right)
\end{array}\right) .
$$

Тогда при тех же условиях на $x$ для компонент матрицы $Z$ имеем

$$
Z_{11} O\left(x^{i \nu_{0}}\right)+Z_{12} O\left(x^{-i \nu_{0}}\right) \sim O\left(x^{i \nu_{0}-1}\right)+O\left(x^{i \nu_{0}+1}\right)
$$

и

$$
Z_{11} O\left(x^{i \nu_{0}}\right)+Z_{12} O\left(x^{-i \nu_{0}}\right) \sim o\left(x^{i \nu_{0}+1}\right)+o\left(x^{i \nu_{0}+1}\right) .
$$

Очевидно, что при рассматриваемых $x$ должно выполняться соотношение $Z_{12}=$ $o\left(Z_{11}\right)$. Отсюда, в свою очередь, заключаем, что $Z_{11}$ линейна, а $Z_{12}$ постоянна. Аналогично при $x \rightarrow+\infty$

$$
G_{-} \sim\left(\begin{array}{cc}
O\left(x^{i \nu_{0}}\right) & O\left(x^{i \nu_{0}}\right) \\
o\left(x^{-i \nu_{0}}\right) & O\left(x^{-i \nu_{0}}\right)
\end{array}\right) \quad \text { и } \quad G_{-}^{\prime} \sim\left(\begin{array}{cc}
O\left(x^{i \nu_{0}-1}\right) & O\left(x^{i \nu_{0}+1}\right) \\
o\left(x^{-i \nu_{0}+1}\right) & O\left(x^{-i \nu_{0}-1}\right)
\end{array}\right) .
$$

Тогда при тех же $x$

$$
Z_{21} O\left(x^{i \nu_{0}}\right)+Z_{22} O\left(x^{-i \nu_{0}}\right) \sim o\left(x^{i \nu_{0}-1}\right)+o\left(x^{-i \nu_{0}+1}\right)
$$

и

$$
Z_{21} O\left(x^{i \nu_{0}}\right)+Z_{22} O\left(x^{-i \nu_{0}}\right) \sim O\left(x^{-i \nu_{0}-1}\right)+O\left(x^{-i \nu_{0}+1}\right) .
$$

Отсюда, как и выше, делаем вывод о том, что $Z_{21}$ постоянна, а $Z_{22}$ линейна. Возвращаясь к равенству (21), мы получаем $Z$ в виде $Z=-i \frac{x}{2} \sigma_{3}+C$, где $C$ - постоянная матрица. Это наблюдение позволяет переписать равенство (21) в виде $G_{ \pm}^{\prime}-i \frac{x}{2}\left[G_{ \pm}, \sigma_{3}\right]=C G_{ \pm}$, где квадратные скобки обозначают коммутатор матриц. Диагональные элементы матрицы левой части этого равенства ведут себя как $O\left(x^{ \pm i \nu_{0}-1}\right)$ на бесконечности. Следовательно, $C=\left(\begin{array}{ll}0 & \lambda \\ \mu & 0\end{array}\right)$. Отсюда $G_{12}^{\prime}+i x G_{12}=\lambda G_{22}, G_{22}^{\prime}=\mu G_{12}$, и тогда

$$
G_{12}^{\prime \prime}+i x G_{12}^{\prime}+i G_{12}-\lambda \mu G_{12}=0 .
$$

Если положить $G_{12}=u e^{-i \frac{x^{2}}{4}}$, то для $u$ мы получим уравнение

$$
u^{\prime \prime}+\frac{x^{2}}{4} u=-i\left(\frac{1}{2}+\nu\right) u, \quad \nu=i \lambda \mu,
$$

где $\nu$ является пока не известным параметром.

Решения этого уравнения называют функциями параболического цилиндра или функциями Вебера. Используя стандартные обозначения для функций Вебера $D_{\nu}$, мы запишем решения $u$ в виде $u=D_{\nu}\left( \pm e^{i \frac{\pi}{4}} x\right)$. При этом при подходящем выборе констант $C_{1}, C_{2}$

$$
\left(G_{-}\right)_{12}=e^{-i \frac{x^{2}}{4}}\left(C_{1} D_{\nu}\left(e^{i \frac{\pi}{4}} x\right)+C_{2} D_{\nu}\left(-e^{i \frac{\pi}{4}} x\right)\right) .
$$

Аналогично, $G_{21}^{\prime}-i x G_{21}=\mu G_{11}, G_{11}^{\prime}=\lambda G_{21}$ и, следовательно,

$$
G_{21}^{\prime \prime}-i x G_{21}^{\prime}-i G_{21}-\lambda \mu G_{21}=0 .
$$

Полагая $G_{21}=v e^{i x^{2} / 4}$, для $v$ получим уравнение

$$
v^{\prime \prime}+\frac{x^{2}}{4} v=i\left(\frac{1}{2}-\nu\right) v,
$$

где $\nu$ то же, что и выше. 
Через функции Вебера решения этого уравнения запишутся в виде $v=$ $D_{\nu-1}\left( \pm e^{i \frac{\pi}{4}} x\right)$; при этом

$$
\left(G_{-}\right)_{21}=e^{i \frac{x^{2}}{4}}\left(E_{1} D_{\nu-1}\left(e^{i \frac{\pi}{4}} x\right)+E_{2} D_{\nu-1}\left(-e^{i \frac{\pi}{4}} x\right)\right),
$$

опять же при подходящем выборе констант $E_{1}, E_{2}$.

Aсимптотика функиий Вебера. Для определения констант нам потребуются асимптотики функций Вебера, которые хорошо известны и могут быть найдены, например, в [1]. При $x \rightarrow+\infty$

$$
\begin{aligned}
D_{\nu}\left(e^{i \frac{\pi}{4}} x\right)= & \left(e^{i \frac{\pi}{4}} x\right)^{\nu} e^{-i \frac{x^{2}}{4}}\left(1+O\left(x^{-2}\right)\right), \\
D_{\nu}\left(-e^{i \frac{\pi}{4}} x\right)= & \left(e^{-i \frac{3 \pi}{4}} x\right)^{\nu} e^{-i \frac{x^{2}}{4}}\left(1+O\left(x^{-2}\right)\right) \\
& \quad-\frac{\sqrt{2 \pi}}{\Gamma(-\nu)} e^{-\nu \pi i}\left(e^{-i \frac{3 \pi}{4}} x\right)^{-\nu-1} e^{i \frac{x^{2}}{4}}\left(1+O\left(x^{-2}\right)\right),
\end{aligned}
$$

а при $x \rightarrow-\infty$

$$
\begin{aligned}
& D_{\nu}\left(e^{i \frac{\pi}{4}} x\right)=\left(e^{-i \frac{3 \pi}{4}}|x|\right)^{\nu} e^{-i \frac{x^{2}}{4}}\left(1+O\left(x^{-2}\right)\right) \\
&-\frac{\sqrt{2 \pi}}{\Gamma(-\nu)} e^{-\nu \pi i}\left(e^{-i \frac{3 \pi}{4}}|x|\right)^{-\nu-1} e^{i \frac{x^{2}}{4}}\left(1+O\left(x^{-2}\right)\right), \\
& D_{\nu}\left(-e^{i \frac{\pi}{4}} x\right)=\left(e^{i \frac{\pi}{4}}|x|\right)^{\nu} e^{-i \frac{x^{2}}{4}}\left(1+O\left(x^{-2}\right)\right) .
\end{aligned}
$$

Следует обратить внимание на порядок поправочных членов разложения.

Определение констант. Константы определяются, исходя из асимптотического поведения матрицы $G$, см. (20). При этом мы хотим вернуть зависимость от параметра $t$, для чего в предыдущих соотношениях следует заменить $x$ на $\sqrt{t} x$.

Общее поведение $\left(G_{-}\right)_{12}$ на бесконечности ведет к равенству $\nu=i \nu_{0}$. Далее, при $x \rightarrow-\infty$ мы находим, что $G_{12}$ является величиной порядка $o\left(x^{i \nu_{0}}\right)$ лишь при $C_{2}=-C_{1} e^{-i \pi \nu}$, и тогда при $x \rightarrow+\infty$ мы получаем

$$
C_{1}\left(1-e^{-2 \pi \nu i}\right)(\sqrt{t})^{\nu} e^{i \frac{\pi}{4} \nu}=-\frac{b_{0}}{\Delta_{0}} e^{-A_{+}}, \quad \text { т. е. } C_{1}=-\frac{a_{0} d_{0}}{c_{0} \Delta_{0}} t^{-i \frac{\nu_{0}}{2}} e^{-A_{+}+\frac{\pi}{4} \nu_{0}} .
$$

Аналогично, $G_{21}$ убывает при $x \rightarrow+\infty$ при условии, что $E_{2}=0$. Устремляя $x \mathrm{k}-\infty$, находим

$$
E_{1} \frac{\sqrt{2 \pi}}{\Gamma\left(1-i \nu_{0}\right)} e^{\frac{\pi \nu_{0}}{4}}(\sqrt{t})^{-i \nu_{0}}=-\frac{c_{0}}{\Delta_{0}} e^{\pi \nu_{0}-B_{+}},
$$

откуда

$$
E_{1}=-\frac{c_{0} \Gamma\left(1-i \nu_{0}\right)}{\Delta_{0} \sqrt{2 \pi}} t^{i \frac{\nu_{0}}{2}} e^{\frac{3 \pi}{4} \nu_{0}-B_{+}} .
$$

Далее, замечаем, что $\left(G_{-}\right)_{22} \sim \tau_{-}$при $x \rightarrow \infty$ и одновременно $G_{22} \sim \frac{i x}{\lambda} G_{12}$ при $x \rightarrow-\infty$, т. е. при $x<0$

$$
\frac{i x}{\lambda} C_{1} \frac{-\sqrt{2 \pi}}{\Gamma\left(-i \nu_{0}\right)} e^{\pi \nu_{0}}\left(|x| e^{-i \frac{3 \pi}{4}}\right)^{-1-i \nu_{0}}=\left(\sqrt{t}|x| e^{i \pi}\right)^{-i \nu_{0}} e^{B_{-}},
$$


откуда

$$
\lambda=\frac{a_{0} d_{0} \sqrt{2 \pi}}{c_{0} \Delta_{0} \Gamma\left(-i \nu_{0}\right)} e^{i \frac{\pi}{4}-\frac{\pi}{2} \nu_{0}-A_{+}-B_{-}} .
$$

Аналогично, $\left(G_{-}\right)_{11} \sim \sigma_{-}$при $x \rightarrow \infty$ и $G_{11} \sim-\frac{i x}{\mu} G_{21}$ при $x \rightarrow+\infty$, т. е. при $x>0$

$$
-\frac{i x}{\mu} E_{1}\left(x e^{i \frac{\pi}{4}}\right)^{i \nu_{0}-1}=\left(\sqrt{t} x e^{2 \pi i}\right)^{i \nu_{0}} e^{A_{-}},
$$

откуда

$$
\mu=\frac{c_{0} \nu_{0} \Gamma\left(-i \nu_{0}\right)}{\Delta_{0} \sqrt{2 \pi}} e^{-i \frac{\pi}{4}+\frac{5 \pi}{2} \nu_{0}-A_{-}-B_{+}} .
$$

Это же значение, для контроля вычислений, ввиду равенства $e^{A_{+}+A_{-}+B_{+}+B_{-}}=$ $\Delta_{0}^{-1}$ мы получим из соотношения $\lambda \mu=\nu_{0}$.

2.4. Асимптотика матрицы $\boldsymbol{H}_{-}$. Асимптотика при $t \rightarrow+\infty$ матрицы $H_{-}$ теперь определяется равенством

$$
H_{-} \sim G_{-}\left(\begin{array}{cc}
\sigma_{-}^{-1} & 0 \\
0 & \tau_{-}^{-1}
\end{array}\right) L_{-}^{-1}\left(\begin{array}{cc}
\alpha_{-} & 0 \\
0 & \beta_{-}
\end{array}\right) .
$$

Например, в области значений величины $|x|$, исключающей окрестность нуля,

$$
H_{-} \sim\left(\begin{array}{cc}
\alpha_{-}+\alpha_{-} \tau_{-}^{-1} G_{12} \delta_{0} T Q & \beta_{-} \tau_{-}^{-1} G_{12}+\beta_{-} \gamma_{0} T^{-1} P \\
\alpha_{-} \sigma_{-}^{-1} G_{21}+\alpha_{-} \delta_{0} T Q & \beta_{-}+\beta_{-} \sigma_{-}^{-1} G_{21} \gamma_{0} T^{-1} P
\end{array}\right) .
$$

Строгое обоснование этого соотношения будет закончено в следующем разделе.

Здесь же выпишем явно старший член этой асимптотики при больших $|x|$ для элемента $\left(H_{-}\right)_{12}$. Именно эта асимптотика как раз наиболее важна в приложениях к нелинейным уравнениям математической физики. Легко видеть, что эту асимптотику проще выделить, устремляя $x \rightarrow-\infty$. В этом случае

$$
\left(H_{-}\right)_{12} \sim \tau_{-}^{-1} G_{12}=\frac{a_{0} d_{0}}{c_{0} \Delta_{0}} \frac{\sqrt{2 \pi}}{\Gamma\left(-i \nu_{0}\right)} t^{-1 / 2-i \nu_{0}} e^{-A_{+}-B_{-}} e^{-i \frac{\pi}{4}} e^{-\frac{\pi \nu_{0}}{2}} \cdot \frac{1}{x} .
$$

2.5. Обоснования. 2.5.1. Исходная позииия. Напомним, что решение модельной задачи $G_{+}=G_{-} V_{0}$ привело нас лишь к приближенной аналитической факторизации вида

$$
\Theta_{-} V \Theta_{+}=\left(L_{-}+\mathscr{L}_{+}\right) \Omega_{-} G_{-}^{-1} G_{+} \Omega_{+}\left(R_{+}+\mathscr{R}_{-}\right),
$$

где $\mathscr{L}_{+}=p(L)-I$ и $\mathscr{R}_{-}=q(R)-I$ малы при $t \rightarrow \infty$. А именно, как это сразу следует из соотношений (10), (13), с точностью до величин порядка $O\left(t^{-\infty}(1+\right.$ $\left.|x|)^{-1}\right)$,

$$
\mathscr{L}_{+}=t^{-i \nu_{0}} \sum_{\substack{k \geqslant 1 \\ 0 \leqslant j \leqslant k}} l_{k j+}(\sqrt{t} x) t^{-k / 2} \ln ^{j} t, \quad \mathscr{R}_{-}=t^{i \nu_{0}} \sum_{\substack{k \geqslant 1 \\ 0 \leqslant j \leqslant k}} r_{k j+}(\sqrt{t} x) t^{-k / 2} \ln ^{j} t,
$$

где матричные функции $l_{k l+}$ и $r_{k l-}$ принадлежат тому же классу гладкости и убывания, что и функции $\varphi_{k l \pm}, \psi_{k l \pm}$, см. формулы (14), (15). Полагая $S_{+}=$ $G_{+} \Omega_{+} R_{+} \Theta_{+}^{-1}, S_{-}=G_{-} \Omega_{-}^{-1} L_{-}^{-1} \Theta_{-}$, мы перепишем $(25)$ в виде $\left(S_{+}+\mathscr{E}_{1}\right)=$ $\left(S_{-}+\mathscr{E}_{2}\right) V$, где матрицы $\mathscr{E}_{1}=G_{+} \Omega_{+} \mathscr{R}_{-} \Theta_{+}^{-1}, \mathscr{E}_{2}=G_{-} \Omega_{-}^{-1}\left[\left(L_{-}+\mathscr{L}_{+}\right)^{-1}-L_{-}^{-1}\right] \Theta_{-}$ 
малы, но не аналитичны. Для того чтобы доказать, что $S_{ \pm}$действительно являются асимптотиками решений $H_{ \pm}$рассматриваемой задачи сопряжения, полезно вспомнить о связях задачи сопряжения с интегральными уравнениями.

2.5.2. Связи с сингулярными интегральными уравнениями. Для установления этих связей удобно перейти к транспонированной задаче Римана вида

$$
H_{+}^{T}=V^{T} H_{-}^{T}, \quad H_{ \pm}^{T} \underset{x \rightarrow \infty}{\sim} I .
$$

Обозначим через $F$ оператор умножения на матричную функцию $F(x)=$ $I-V$. Через $F^{T}$ будет обозначаться оператор умножения на транспонированную матрицу. Из теории краевых задач известно, что если $H_{ \pm}^{T}$ являются решениями рассматриваемой задачи сопряжения, то для оператора $I-F^{T} q$ существует обратный, равный $\left(I-F^{T} q\right)^{-1}=I+F^{T} H_{-}^{T} q\left(H_{+}^{T}\right)^{-1}$. Действительно,

$$
\begin{gathered}
\left(I-F^{T} q\right)\left(I+F^{T} H_{-}^{T} q\left(H_{+}^{T}\right)^{-1}\right)=I-F^{T} q+F^{T} H_{-}^{T} q\left(H_{+}^{T}\right)^{-1}-F^{T} q F^{T} H_{-}^{T} q\left(H_{+}^{T}\right)^{-1} \\
=I-F^{T} q+F^{T} H_{-}^{T} q\left(H_{+}^{T}\right)^{-1}-F^{T} q\left(H_{-}^{T}-H_{+}^{T}\right) q\left(H_{+}^{T}\right)^{-1}=I,
\end{gathered}
$$

и аналогичный результат получается при перемножении этих операторов в обратном порядке.

Наоборот, пусть оператор $I-F^{T} q$ имеет обратный. Определим матричную функцию $K^{T}$ как решение уравнения $\left(I-F^{T} q\right) K^{T}=F^{T}$. Тогда непосредственно проверяется, что транспонированная задача сопряжения имеет решение вида $H_{+}^{T}=I-p\left(K^{T}\right), H_{-}^{T}=I+q\left(K^{T}\right)$. Действительно,

$$
\begin{aligned}
\left(I-F^{T}\right)\left(I+q\left(K^{T}\right)\right) & =I-F^{T}+q\left(K^{T}\right)-F^{T} q\left(K^{T}\right) \\
& =I-p\left(K^{T}\right)-F^{T}+\left(I-F^{T} q\right) K^{T}=I-p\left(K^{T}\right) .
\end{aligned}
$$

Как следствие получим $H_{+}=I-p(K), H_{-}=I+q(K)$.

2.5.3. Сведение $к$ интегралъным уравнениям. Положим

$$
\mathscr{E}=\Theta_{-}^{-1} \mathscr{L}_{+} W_{0} R_{+} \Theta_{+}^{-1}+\Theta_{-}^{-1} L_{-} W_{0} \mathscr{R}_{-} \Theta_{+}^{-1}+\Theta_{-}^{-1} \mathscr{L}_{+} W_{0} \mathscr{R}_{-} \Theta_{+}^{-1},
$$

где $W_{0}=\Omega_{-} V_{0} \Omega_{+}$, см. также (19). Подстановка в правую часть данного равенства разложений (26) приводит нас к разложению $\mathscr{E}$ по порядкам $t^{ \pm i \nu_{0}-k} \ln ^{j} t$ $(0 \leqslant j \leqslant k)$ с коэффициентами, хотя и зависящими от $t$, но ограниченными. При этом $\mathscr{E}=O\left(t^{\left|\operatorname{Im} \nu_{0}\right|-1} \ln t\right)=o(1)$.

Как описывалось выше, пара факторизационных множителей $S_{ \pm}$определяет решение $K_{0}^{T}$ уравнения

$$
\left(I-F_{0}^{T} q\right) K_{0}^{T}=F_{0}^{T}, \quad F_{0}=S_{+}^{-1} S_{-} .
$$

При этом $V=I+F_{0}+\mathscr{E}$. Уравнение на $K^{T}$

$$
\left(I+F_{0}^{T} q+\mathscr{E}^{T} q\right) K^{T}=F_{0}^{T}+\mathscr{E}^{T}
$$

с учетом (28) допускает решение итерациями. В $L_{2}(\mathbb{R})$, в связи с ограниченностью оператора $\left(I+F_{0}^{T} q\right)^{-1}$, это приведет нас к разложению $K$ по порядкам $t^{ \pm i \nu_{0}-k} \ln ^{j} t$ с ограниченными коэффициентами, зависящими от $t$.

Однако для приложений к нелинейным уравнениям математической физики факторизации в классе интегрируемых функций не достаточно. К сожалению, оператор типа $I+F_{0}^{T} q$ не является ограниченно обратимым в классе непрерывных функций, и одна из возможностей преодолеть ситуацию - обратиться к 
классам гёльдеровых функций. Именно на этой возможности мы и остановимся.

Как известно, оператор $q$ ограничен в пространствах $H^{\mu, \theta}$ при $0<\mu<1,0 \leqslant$ $\theta<1$ (лемма Привалова). Однако нам будет полезна некоторая модификация этого свойства, доказательство которой мы приведем в приложении А.2: оператор $q$ ограничен как оператор из $H^{\mu, 1+\theta}$ в $H^{\mu, 1}$.

Ограниченные гёльдеровы функции $g$ пространства $H^{\mu}=H^{\mu, 0}$ являются мультипликаторами пространства $H^{\mu, \theta}$ с нормой, равной $H^{\mu}$-норме соответствующей функции. Как следствие $\left\|\left(I+F_{0}^{T} q\right)^{-1}\right\|_{H^{\mu, 1+\theta} \rightarrow H^{\mu, 1}}=O\left(t^{\mu / 2}\right)$ (в связи с наличием быстрой переменной $\sqrt{t} x$ в аргументе функции $\left.F_{0}\right)$. Наконец, заметим, что из формул (26) вытекает оценка для нормы мультипликатоpa $\mathscr{E}^{T}:\left\|\mathscr{E}^{T}\right\|_{H^{\mu, 1} \rightarrow H^{\mu, 1+\theta}}=O\left(t^{(1-\theta)\left(\left|\operatorname{Im} \nu_{0}\right|-1+\mu / 2\right)}\right)$. Поскольку $\mu$ и $\theta$ могут быть сделаны сколь угодно малыми, то оператор $\mathscr{E}^{T}\left(I+F_{0}^{T} q\right)^{-1}$ является ограниченным оператором малой нормы порядка $O\left(t^{(1-\theta)\left(\left|\operatorname{Im} \nu_{0}\right|-1+\mu\right)}\right)=o(1)$ в пространстве $H^{\mu, 1+\theta}$, что позволяет от уравнения (29) перейти к итерациям в уравнении

$$
\left(I+\mathscr{E}^{T}\left(I+F_{0}^{T} q\right)^{-1}\right) X=\mathscr{E}^{T}\left(I-K_{0}^{T}\right)
$$

для величины $X=\left(I+F_{0}^{T} q\right)\left(K^{T}-K_{0}^{T}\right)$. Как следствие невязка $K^{T}-K_{0}^{T}$ оценивается величиной $O\left(t^{\left|\operatorname{Im} \nu_{0}\right|-1+\varepsilon}\right)$, где $\varepsilon>0$ можно выбрать произвольно малым. Этот факт с учетом неравенства $\left|\operatorname{Im} \nu_{0}\right|<1 / 2$ обеспечивает невязке малость высшего порядка по сравнению со старшим членом $K_{0}^{T}$.

Итак, нами доказана

Теорема 2.2. При достаточно больиих $t>0$ решение рассматриваемой задачи сопряжения (при наложенных ограничениях на коэфбиииенты матрииь сопряэсения) существует и имеет асимптотику, определенную соотношениями $(22)-(24)$ и невязкой $O\left(t^{\left|\operatorname{Im} \nu_{0}\right|-1+\varepsilon}\right)$ относительно $H^{\mu, 1}(\mathbb{R})$, где $\mu>0$ достаточно мало.

\section{§3. Приложение к НУШ}

3.1. Асимптотика решений задачи Коши для НУШ на больших временах. Постановка задачи. Рассматриваемое нелинейное уравнение Шрёдингера имеет вид

$$
i u_{t}+u_{x x}-2|u|^{2} u=0 .
$$

Оно рассматривается в предположении, что $\left.u\right|_{t=0}-$ гладкая и быстро убывающая функция при $x \rightarrow \infty$. Наибольший интерес представляет асимптотическое поведение решения $u$ при $t \rightarrow \infty$ в той области, которая, как известно, контролирует законы сохранения: $0<C_{1} \leqslant|x / t| \leqslant C_{2}<\infty$.

Матричная задача сопряжения, ассоциированная с нелинейным уравнением Шрёдингера, имеет стандартный, описанный выше вид

$$
H_{+}=H_{-} V, \quad V(k)=\left(\begin{array}{cc}
1-|r(k)|^{2} & -\overline{r(k)} e^{-i t \Phi(k)} \\
r(k) e^{i t \Phi(k)} & 1
\end{array}\right),
$$

и соответствует фазовой функции $\Phi(k)=4 k^{2}+2 l k$, где параметр $l$ имеет смысл отношения $x / t$. Функция $r-$ коэффициент отражения - конструируется по 
начальным данным для нелинейного уравнения Шрёдингера, а решение уравнения $u$ восстанавливается, см., например, [7], по решению задачи сопряжения в виде

$$
u(x, t)=2 i \lim _{k \rightarrow \infty} k H_{12}(k, t) .
$$

Здесь $H_{12}$ является соответствующим элементом любой из матриц $H_{ \pm}$.

Замена переменной. Нетрудно заметить, что замена переменной $k \mapsto z=$ $\sqrt{2}(2 k+l / 2)$, позволяет считать фазовую функцию равной $\Phi(z)=z^{2} / 2$. При этом искомое решение нелинейного уравнения Шрёдингера запишется в виде

$$
u(x, t)=\frac{i}{\sqrt{2}} e^{i x^{2} /(4 t)} \lim _{z \rightarrow \infty} z H_{12}(z, t) .
$$

Acuмnтотика $H_{12}$. Достаточно воспользоваться результатом (24), полагая $a=1-|r|^{2}, b=-\bar{r}, c=r, d=1, \Delta=1$. Здесь все функции рассматриваются от преобразованной переменной.

Положим

$$
B=\frac{1}{2 \pi} \int_{-\infty}^{0} \ln |y| d \ln \left(1-|r(y)|^{2}\right), \quad \nu_{0}=\frac{-1}{2 \pi} \ln \left(1-\left|r_{0}\right|^{2}\right), r_{0}=r(0) .
$$

Отметим, что

$\ln \sigma_{+}(x) \underset{x \rightarrow 0}{\sim}-i \nu_{0} \ln (x+i 0)+\frac{1}{2 \pi i} \int_{-\infty}^{0} \ln (-y+i 0) d \ln \left(1-|r(y)|^{2}\right)=-i \nu_{0} \ln (x+i 0)-i B$, откуда $A_{+}=-i B$. Аналогично,

$$
\begin{array}{r}
-\ln \tau_{-}(x) \underset{x \rightarrow 0}{\sim} i \nu_{0} \ln (x-i 0)-\frac{1}{2 \pi i} \int_{-\infty}^{0} \ln (-y-i 0) d \ln \left(1-|r(y)|^{2}\right) \\
=i \nu_{0} \ln (x-i 0)+i B+2 \pi \nu_{0},
\end{array}
$$

откуда $B_{-}=-i B-2 \pi \nu_{0}$. Значит, при $x, t \rightarrow \infty$

$$
H_{12} \sim \frac{1}{r_{0}} \frac{\sqrt{2 \pi}}{\Gamma\left(-i \nu_{0}\right)} t^{-1 / 2-i \nu_{0}} e^{2 i B} e^{-i \pi / 4} e^{-\pi \nu_{0} / 2} \cdot \frac{1}{x}
$$

где мы воспользовались равенством $a_{0}=e^{-2 \pi \nu_{0}}$. Теперь мы можем выписать асимптотику решения нелинейного уравнения Шрёдингера.

Асимптотика решения НУШ. В исходных терминах с учетом (30)

$$
\begin{aligned}
& u=A e^{i \Psi}, \quad A=\sqrt{\frac{\nu_{0}}{2 t}}, \quad \nu_{0}=\frac{-1}{2 \pi} \ln \left(1-\left|r_{0}\right|^{2}\right), \quad r_{0}=r\left(-\frac{x}{4 t}\right), \\
& \Psi=\frac{x^{2}}{4 t}-\nu_{0} \ln t+\arg \Gamma\left(i \nu_{0}\right)-\arg r_{0} \\
& \quad+\frac{1}{\pi} \int_{-\infty}^{-x /(4 t)} \ln \left(-\frac{x}{4 t}-k\right) d \ln \left(1-|r(k)|^{2}\right)-\nu_{0} \ln 8+\frac{\pi}{4} .
\end{aligned}
$$

Эта асимптотика была впервые получена, хотя и не строго, в работе [8].

3.2. Заметки по сравнению подходов. Авторы работ [6], [7] назвали свой подход методом наибыстрейшего спуска для задачи Римана с осциллирующими 
матрицами. Основная часть их вычислений состоит из решения серии вспомогательных задач Римана на крестовидных контурах. Идея действительно заимствована из метода перевала. Но отношения между упомянутыми задачами Римана довольно хитроумные. Трудности подхода Дейфта-Жу предсказуемы. Необходимым условием применимости метода перевала для вычисления асимптотики интеграла $\int_{\Gamma} F(z) e^{i t \Phi(z)} d z$ является аналитичность функций $F$ и $\Phi$. И хотя замена переменных спасает положение при неаналитичности функции $\Phi$, неаналитичность функции $F$ является преградой, для преодоления которой требуются дополнительные ухищрения.

Выбор в пользу метода стационарной фазы, сделанный нами, улучшает ситуацию.

\section{А. Приложение}

А.1. Доказательство теоремы 2.1. Остановимся для определенности на доказательстве разложения (10). Пусть $\chi(x)$ будет бесконечно дифференцируемой функцией, равной тождественно нулю в окрестности нуля и единице в окрестности бесконечности. Рассмотрим интеграл

$$
\begin{aligned}
& \frac{-1}{2 \pi i} \int_{0}^{\infty} \frac{\gamma_{\infty}(y) \chi(y) e^{-i t y^{2}} d y}{x}-y+i 0 \\
& \quad=\frac{-1}{2 \pi i} \int_{0}^{\infty} \frac{\gamma(y) \chi(y) e^{-i t y^{2}} d y}{x-y+i 0}-\frac{-1}{2 \pi i} \int_{0}^{\infty} \frac{\gamma_{0}(y) \chi(y) e^{-i t y^{2}} d y}{x-y+i 0} .
\end{aligned}
$$

Поскольку вне окрестности нуля функция $\gamma$ является гладкой и быстро исчезающей на бесконечности вместе с производными, стандартная техника метода стационарной фазы легко ведет к оценке первого слагаемого в правой части формулы (32):

$$
\frac{-1}{2 \pi i} \int_{0}^{\infty} \frac{\gamma(y) \chi(y) e^{-i t y^{2}} d y}{x-y+i 0}=O\left(t^{-\infty}(1+|x|)^{-1}\right) .
$$

Второе слагаемое с точностью до постоянного множителя приводится к интегралу

$$
\frac{-1}{2 \pi i} \int_{0}^{\infty} \frac{(y-i 0)^{2 i \nu_{0}} \chi(y) e^{-i t y^{2}} d y}{x-y+i 0}
$$

оценка которого облегчается деформацией контура интегрирования. А именно, часть полуоси, на которой $\chi(y)=1$, можно продеформировать в нижнюю комплексную полуплоскость, совмещая с частью луча $\arg y=-\pi / 4$ (согласно лемме Жордана, такая деформация возможна при $\left.\operatorname{Re}\left(i \nu_{0}\right)<1\right)$. После описанной деформации оценка интеграла элементарна, поскольку на контуре интегрирования нет точки перевала. Итак,

$$
\frac{-1}{2 \pi i} \int_{0}^{\infty} \frac{\gamma_{0}(y) \chi(y) e^{-i t y^{2}} d y}{x-y+i 0}=O\left(t^{-\infty}(1+|x|)^{-1}\right)
$$

и как следствие

$$
\frac{-1}{2 \pi i} \int_{0}^{\infty} \frac{\gamma_{\infty}(y) \chi(y) e^{-i t y^{2}} d y}{x-y+i 0}=O\left(t^{-\infty}(1+|x|)^{-1}\right)
$$


Пусть теперь $\chi_{0}=1-\chi$. Рассмотрим интеграл

$$
\frac{-1}{2 \pi i} \int_{0}^{\infty} \frac{\gamma_{\infty}(y) \chi_{0}(y) e^{-i t y^{2}} d y}{x-y+i 0} .
$$

Разлагая функцию $\gamma_{\infty}$ в ряд в окрестности нуля, см. (9), рассмотрим следующий вклад в рассматриваемый интеграл:

$$
\frac{-1}{2 \pi i} \int_{0}^{\infty} \frac{y^{\varkappa} \ln ^{m} y \chi_{0}(y) e^{-i t y^{2}} d y}{x-y+i 0},
$$

где $\varkappa=2 i \nu_{0}+k, 0 \leqslant m \leqslant k, k \geqslant 1$. На участке, где $\chi_{0}(y)=1$, мы можем продеформировать контур интегрирования в нижнюю комплексную полуплоскость к началу луча $\arg y=-\pi / 4$. Полученный интеграл мы запишем как разность

$$
\frac{-1}{2 \pi i} \int_{\arg y=-\pi / 4} \frac{y^{\varkappa} \ln ^{m} y e^{-i t y^{2}} d y}{x-y}-\frac{-1}{2 \pi i} \int_{\Gamma} \frac{y^{\varkappa} \ln ^{m} y \chi_{0}(y) e^{-i t y^{2}} d y}{x-y+i 0},
$$

где контур $\Gamma$ состоит из части луча $\arg y=-\pi / 4$, не содержащей окрестности нуля, отрезка вещественной оси, на котором $\chi_{0}(y) \neq 1$, и дуги, соединяющей только что описанные части контура $\Gamma$, а именно вершину луча и левый конец отрезка. На невещественной части контура $\Gamma$ функция $\chi_{0}$ полагается тождественно равной единице.

Оценка интеграла по Г элементарна и ведет (как и выше) к равенству

$$
\frac{-1}{2 \pi i} \int_{\Gamma} \frac{y^{\varkappa} \ln ^{m} y \chi_{0}(y) e^{-i t y^{2}} d y}{x-y+i 0}=O\left(t^{-\infty}(1+|x|)^{-1}\right) .
$$

В первом интеграле в (35) мы сделаем замену переменной $\sqrt{t} y=z e^{-i \pi / 4}$, что приведет нас к равенству

$$
\begin{aligned}
\frac{-1}{2 \pi i} \int_{\arg y=-\pi / 4} \frac{y^{\varkappa} \ln ^{m} y e^{-i t y^{2}} d y}{x-y} & =\frac{-t^{-\varkappa / 2}}{2 \pi i} \int_{0}^{\infty} \frac{z^{\varkappa}\left(\ln z-\frac{1}{2} \ln t-i \frac{\pi}{4}\right)^{m} e^{-z^{2}-i \frac{\pi}{4} \varkappa} d z}{e^{i \frac{\pi}{4}} \sqrt{t} x-z} \\
& =t^{-\varkappa / 2} \sum_{0 \leqslant 1 \leqslant m} \ln ^{l} t f_{\varkappa m l}(\sqrt{t} x),
\end{aligned}
$$

где функции $f_{\varkappa m l}$ определяются соответствующими интегралами, получающимися при вычленении степени логарифма $\ln ^{l} t$.

Легко видеть, что при фиксированном $\nu_{0}$ функции $\varphi_{k j+}$ определяются равенствами $\varphi_{k j+}=\sum_{j \leqslant m \leqslant k} f_{\varkappa m j}$. Их свойства очевидны.

Для завершения доказательства следует оценить погрешность разложения, возникающую при учете лишь конечного (но достаточно большого) числа слагаемых разложения $\gamma_{\infty}$ в $(9)$, т. е. интеграл вида

$$
\frac{-1}{2 \pi i} \int_{0}^{\infty} \frac{\Phi(y) \chi_{0}(y) e^{-i t y^{2}} d y}{x-y+i 0}
$$

где $\Phi$ - гладкая (достаточное количество раз дифференцируемая) функция, равная $O\left(y^{N}\right)$ при $y \rightarrow 0$, причем $N$ можно считать сколь угодно большим. Без ограничения общности можно считать, что $\Phi(y)=y^{2 M+1} \Psi(y)$, где $M$ сколь 
угодно велико (вместе с $N)$ и $\Psi$ - сколь угодно гладкая функция. Тогда по аналогии с предыдущим

$$
\begin{aligned}
& \frac{-1}{2 \pi i} \int_{0}^{\infty} \frac{\Phi(y) \chi_{0}(y) e^{-i t y^{2}} d y}{x-y+i 0}=\frac{-1}{2 \pi i} \int_{0}^{\infty} \frac{\left(\Psi(y)-\Psi(x) \chi_{1}(x)\right) y^{2 M+1} \chi_{0}(y) e^{-i t y^{2}} d y}{x-y} \\
+ & \frac{-\Psi(x) \chi_{1}(x)}{2 \pi i} \int_{0}^{\infty} \frac{y^{2 M+1} \chi_{0}(y) e^{-i t y^{2}} d y}{x-y+i 0}=O\left(t^{-M}(1+|x|)^{-1}\right)+O\left(t^{-M}(1+|x|)^{-1}\right),
\end{aligned}
$$

где $\chi_{1}$ - гладкая финитная функция, тождественно равная единице на носителе функции $\chi_{0}$.

А.2. Доказательство ограниченности оператора $q: H^{\mu, 1+\theta} \rightarrow H^{\mu, 1}$. Будем считать, что $x \rightarrow+\infty$. Введем разбиение единицы: $1=\sum_{i=1}^{4} \chi_{i}$, где $\chi_{i}-$ гладкие функции со значениями на интервале $[0,1]$ и носителями, соответственно равными $(-\infty, x / 2+1],[x / 2, x-1],[x-2, x+2],[x+1,+\infty)$, и рассмотрим интеграл

$$
\int_{-\infty}^{+\infty} \frac{\varphi(y) d y}{x-y-i 0}=\sum_{i=1}^{4} \int_{-\infty}^{+\infty} \frac{\chi_{i}(y) \varphi(y) d y}{x-y-i 0}=\sum_{i=1}^{4} I_{i}
$$

где $\varphi \in H^{\mu, 1+\theta}, 0<\mu<1,0<\theta<1$. Норму функции $\varphi$ в $H^{\mu, 1+\theta}$ будем обозначать через $\|\varphi\|$. Отметим, что сингулярным является только интеграл $I_{3}$.

Оценки интегралов $I_{1}$ и $I_{2}$ элементарны: $\left|I_{1}\right| \leqslant C_{1}(1+|x|)^{-1}\|\varphi\|,\left|I_{2}\right| \leqslant C_{2}(1+$ $|x|)^{-1}\|\varphi\|$. Оценку интеграла $I_{4}$ легко провести, используя неравенство Гёльдера

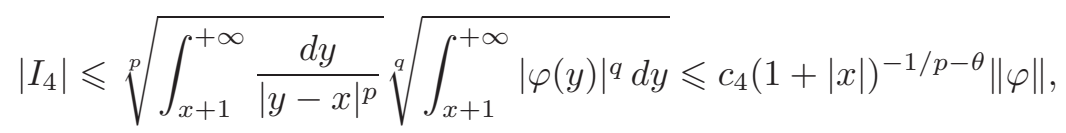

где $p$ и $q$ - сопряженные показатели, и положив затем $q=\theta^{-1}$. Оценка интеграла $I_{3}$ проводится стандартно для сингулярного интеграла:

$$
\left|I_{3}\right| \leqslant \int_{x-2}^{x+2}\left|\frac{\varphi(y)-\varphi(x)}{x-y}\right| d y+\left|\varphi(x) \int_{x-2}^{x+2} \frac{\chi_{3}(y) d y}{x-y-i 0}\right| \leqslant C_{3}(1+|x|)^{-1-\theta}\|\varphi\| .
$$

Оценка гёльдеровости интегралов $I_{i}$ проводится аналогично.

\section{ЛитерАТУРА}

[1] Г. Бейтмен, А. Эрдейи, Высшие трансцендентные функции, $m$. 2, Справочная математическая библиотека, Наука, М., 1966.

[2] А. М. Будылин, В. С. Буслаев, Квазиклассические интегральные уравнения $и$ асимптотическое поведение решений уравнения Кортевега-де Фриза при больших временах, Докл. РАН, 348:4 (1996), 455-458.

[3] А. М. Будылин, В. С. Буслаев, Уравнение Гелъфанда-Левитана-Марченко и асимптотическое поведение решений нелинейного уравнения Шрёдингера при больших временах, Алгебра и анализ, 12:5 (2000), 64-105.

[4] И. Ц. Гохберг, Н. Я. Крупник, Введение в теорию одномерных сингулярных интегралъных операторов, Штиинца, Кишинев, 1973.

[5] А. Р. Итс, Асимптотика решений нелинейного уравнения Шрёдингера и изомонодромные деформации систем линейных дифференциальных уравнений, Докл. АН CCCP, 261:1 (1981), 14-18.

[6] P. A. Deift, X. Zhou, A steepest descent method for oscillatory Riemann-Hilbert problems. Asymptotics for the MKdV equation, Ann. of Math., 137:2 (1993), 295-368. 
[7] P. A. Deift, X. Zhou, Long-time behavior of the non-focusing nonlinear Schrödinger equation-a case study, University of Tokyo, Tokyo, 1994.

[8] В. Е. Захаров, С. В. Манаков, Асиптотическое поведение нелинейных волновых систем, интегрируемых методом обратной задачи рассеяния, ЖЭТФ, 71:1 (1976), 203-215.

Санкт-Петербургский государственный университет budylin@math.nw.ru

Поступило в редакцию 23 марта 2012 г. 